\title{
DUAL-POLARIZED TERRASAR-X DATA FOR OIL-SPILL OBSERVATION
}

\author{
D. Velotto ${ }^{(1)}$, M. Migliaccio ${ }^{(2)}$, F. Nunziata ${ }^{(2)}$, S. Lehner ${ }^{(1)}$ \\ ${ }^{(1)}$ DLR, German Aerospace Center, Remote Sensing Technology Institute \\ Oberpfaffenhofen, 82234 - Weßling, Germany \\ ${ }^{(2)}$ Università di Napoli Parthenope, Dipartimento per le Tecnologie \\ Centro Direziale, isola C4 - 800143 - Napoli, Italy
}

\begin{abstract}
The Synthetic Aperture Radar (SAR) is unanimously recognized as the most important remote sensing tool for sea oil spill monitoring. The TerraSAR-X satellite can provide highquality, multi-mode X-band SAR-data from Ocean surface for scientific research and associated applications. For the purposes of this study Single Look Slant Range Complex (SSC) Dual Polarization StripMap (SM) TerraSAR-X products are employed.

Physically based polarimetric approaches, aimed at exploiting fully the information carried on the scattered waves, have been shown to be useful for both observing oil spills and distinguishing them from biogenic look-alikes [1]-[2]. Following this, the phase difference between the complex $\mathrm{HH}$ and VV channels, which has been demonstrated to be suitable to observe sea oil spills in C-band SAR data [2], will be employed to distinguish in physical terms the X-band signal scattered off the slick-free and slick-covered sea surface as images as collected by the new TerraSAR-X satellite.

Experiments, accomplished over a meaningful set of SSC dual-polarized TerraSAR-X data in which both oil and look-alikes are present, show that, for both observing sea oil slicks and distinguishing them from weak-damping look-alikes in X-band polarimetric SAR data, the standard deviation of the phase difference is the key information.
\end{abstract}

\section{Introduction}

A synoptic sea oil spill monitoring, based on remotely sensed data, is fundamental for three mainly reasons [3]:

- To contrast illegal vessel discharge (the most important source of sea oil pollution)

- To have an early warning system in case of environmental disaster (e. g. tanker accidents)

- To assist law enforcements (on global, regional and local scale) 
Although there are many remote sensing equipments and techniques available for this purpose, microwave sensors are often preferred to optical sensors. The SAR is unanimously recognized as the key sensor for this application [4]-[5]:

$\checkmark$ Day and Night capabilities

$\checkmark$ Wide area coverage

$\checkmark$ High resolution data

$\checkmark$ Independence from meteorological conditions (e. g. clouds and fog)

It is well known that oil films floating on the sea surface appear as dark patches on radar images. Unfortunately, oil slicks are not the only phenomena that are detected in this way. Dark areas in SAR images can be due to both man made (e.g. oil spill discharge) and natural phenomena (e.g. biogenic slicks, algae bloom, low wind areas, internal waves, rain cell and so on) since both of them dampen the short gravity and capillary waves responsible for the backscattered signal [4],[5]-[6].

The TerraSAR-X satellite can provide high-quality, multi-mode X-band SAR-data from Ocean surface for scientific research and associated applications. In Fig.1 and Fig. 2 (a)-(b) are reported three TSX images in different acquisition mode in which oil spill from diverse sources are depicted. For the purposes of this study Single Look Slant Range Complex (SSC) Dual Polarization StripMap (SM) TerraSAR-X product are employed.

Physically based polarimetric approaches, aimed at exploiting fully the information carried on the scattered waves, have been shown to be useful for both observing oil spills and distinguishing them from biogenic look-alikes [1]-[2]. Following this, the phase difference between the complex $\mathrm{HH}$ and VV channels (so called Co-Polar phase Differernce and here after referred with the acronym CPD), which has been demonstrated to be suitable to observe sea oil spills in C-band SAR data [2], will be employed to distinguish in physical terms the Xband signal scattered off the slick-free and slick-covered sea surface as images as collected by the new TerraSAR-X satellite.

Experiments, accomplished over a meaningful set of SSC dual-polarized HH-VV TerraSAR-X data in which both oil and look-alikes are present, show the consistence of the CPD probability density function (pdf) model for both observing sea oil slicks and distinguishing them from look-alikes in X-band polarimetric SAR data. 


\section{The Rationale}

The State of Art in the oil spills detection by SAR data have been mainly developed using single polarization acquisitions. To distinguish oil slick from look-alikes these techniques are usually based on [4]:

- Statistical approach

- Background analyses

- Use of Neural network

- Morphological analysis

- External ancillary information

The main goal of this study is demonstrate the capability of the dual-polarization TerraSAR-X mode for oil spill observation. The phase information retrieved from the X-band TerraSAR-X $\mathrm{HH}$ and VV channels is firstly used for this purpose.

Recent analysis have been demonstrated that the CPD pdf is able both to observe oil spill and distinguish biogenic look-alikes in C-band SAR data [2]. It is the first time that this approach is employed in X-band SAR data for oil spill observation.

For horizontal and vertical linearly polarized electric field, the 2x2 scattering matrix measured by fully polarimetric SAR antenna is:

$$
\mathbf{S}=\left(\begin{array}{ll}
\dot{S}_{h h} & \dot{S}_{h v} \\
\dot{S}_{v h} & \dot{S}_{v v}
\end{array}\right),
$$

that considering a reciprocal medium, using amplitude and phase annotation, can be written as:

$$
\mathbf{S}=e^{j \varphi_{v v}}\left(\begin{array}{cc}
S_{h h} e^{j \varphi_{c}} & S_{h v} e^{j \varphi_{x}} \\
S_{h v} e^{j \varphi_{x}} & S_{v v}
\end{array}\right)
$$

where

$$
\varphi_{x}=\varphi_{h v}-\varphi_{v v}=\varphi_{v h}-\varphi_{v v}
$$

and

$$
\varphi_{c}=\varphi_{h h}-\varphi_{v v}
$$

are the Cross-Polarized phase Difference (XPD) and the CPD, respectively.

Under low to moderate wind conditions, the standard deviation of the CPD pdf, being related to the correlation between the complex $\mathrm{HH}$ and VV channels, can be considered as a measure of the departure from the Bragg scattering. 
In particular, in case of slick-free sea surface, the small-scale roughness is well-described by Bragg mechanism, which is characterized by a high HH-VV correlation and, therefore, a narrow CPD pdf is expected, as demonstrated in [2].

When an oil film is present, due to its strong damping properties, a non-Bragg scattering mechanism is in place, which is characterized by a low HH-VV correlation and, therefore a broaden CPD pdf is expected [2].

From an operational viewpoint, a simple filter based on the CPD standard deviation information, being able to measure the departure from Bragg scattering, is expected to act like an emphasise oil filter respect the surrounding sea. In the case of weak-damping look-alikes, since their different damping properties, which lead to a completely different scattering mechanism and characterised by CPD standard deviation values similar to the surrounding sea ones, a non emphasise filter is expected.

\section{Experiments}

In this section a meaningful X-band SSC SAR data have been processed to show the capability of the TerraSAR-X dual-mode for oil spill observation using polarimetric features.

The data was acquired by the TerraSAR-X sensor (X-band $9.65 \mathrm{GHz}$ ) designed to work in different modes and polarization with different swath coverage, incidence angle and resolution. Polarimetric feature, very short revisit time (11 days repeat orbit) together with the wide area coverage and all-day all-weather microwave sensors capability make TerraSAR-X to a very useful tool for Earth's surface monitoring. The SpotLight-, StripMap- and ScanSARmodes provide high resolution images, $\sim 1 \mathrm{~m}, \sim 3 \mathrm{~m}$ and $\sim 15 \mathrm{~m}$ azimuth resolution respectively. Nominally TerraSAR-X is a right looking systems that in emergency status, due to the satellite roll manoeuvre ability, can operate like left looking system. Thanks to the active phased array antenna technology data acquisition at different incidence angles are possible. The incidence angle range in full performance is $20^{\circ}-45^{\circ}$ for StripMap- and ScanSAR-mode and $20^{\circ}-55^{\circ}$ for SpotLight-mode $\left(15^{\circ}-60^{\circ}\right.$ range is accessible in each mode)[7].

For the purposes of this study Single Look Slant Range Complex (SSC) Dual Polarization StripMap (SM) TerraSAR-X product are employed. The SSC is the basic single look product of the focused radar signal for scientific applications that require the full bandwidth and phase information (data are represented as complex numbers). In StripMap-mode the earth surface is illuminated with a continuous sequence of pulses while the antenna beam is fixed in elevation and azimuth. The result is an image strip of the earth with constant resolution in azimuth direction. The dual polarization mode is implemented by toggling the transmit and/or receive 
polarization between consecutive pulses. The effective pulse repetition frequency (PRF) in each polarimetric channel is thus half of the total PRF [7].

Three TerraSAR-X SSC HH/VV dual polarisation complex images in which both oil slick and look-alikes are present will be considered to proof that the physical polarimetric approach described in the section II is applicable in X-band SAR data for oil spill observation. The results show that is possible not only distinguish oil slicks from the surrounding sea but also from some weak-damping surface slicks (oil look-alikes). In details a total of 6 region of interest (ROI) are processed: three of them containing oil-spill due to, illicit vessel discharge (first data set, one ROI selected) and tanker accident (second data set, three ROI selected), two of them in which three ship wakes are detected (third data set, two ROI selected). The COSAR (Complex SAR) TerraSAR-X files, contain all focused complex SAR data, have been processed using 3x3 moving window to estimate the standard deviation of the two mainly polarimetric parameters: CPD and Co-Polarised Ratio (i.e. HH-VV Normalised Radar Cross Section ratio and here after called CPR).

The first data set is relevant to the acquisition of 28 July 2009, 04:18 UTC in Black Sea. Fig. 3 (a) shows an excerpt of the X-band VV power SAR image (it shows the VV channel only for comparison. The HH channel it does not show to save space) in which an illicit vessel oil discharge is clearly visible. The estimated CPD and CPR standard deviation are shown in grey tones in Fig. 3 (b) and 1(c), respectively.

In this case the CPD standard deviation image clearly show features related to the oil spill (compare Figs. 3 (a) - 3 (b)). In this example is also shown the filtering result applied at the CPR polarimetric parameter and it does not witness any features related to the oil spill. As matter of fact, a visual comparison between Figs. 3 (b) and 3 (c) highlights the truth that the CPR information is not useful for oil spill observation in radar images, thus will no longer taken into account. The theoretical model proposed in [2], which predicts a different scattering mechanism in presence of an oil spill in C-band SAR data, is here applied in Xband to show the consistence of the model proposed. In fact, the result can be quantitatively confirmed analysing the measured CPD pdfs, relevant to both oil slick and the surrounding sea surface (see Fig. 3 (d)) in which the measured oil spill CPD pdf is much broader than the surrounding sea surface one.

The second data set is relevant to the acquisition of 16 November 2007 at 03:52 UTC, 5 days after the shipwrecks in Kerch Strait. On November 11, stormy seas and gale-force winds in the narrow Kerch Strait have smashed a Volganeft-139 Russian oil tanker in two parts, spilling at least 2,000 metric tonnes of fuel oil. This data set is relatively important to try out 
the model as we know the ground truth. Due to very large dark areas three ROI are selected in different places (Northerly, Middle and Southerly) and with different shape (short width and long thin dark areas). In Figs. 4, 5 and 6 are reported the three ROI selected with their CPD standard deviation images and measured CPD pdfs. The results are in total agreement to what formerly experienced (see Figs. 4 (b)-(c), 5 (b)-(c), 6 (b)-(c)). It can be also noted that no influence is highlighted by the different oil spill pattern (see elongated shape in Fig. 6 typically due to a spillage from ship like showed in Fig. 2 (b)).

The third data set is relevant to the acquisition of 5 June 2009 at 05:19 UTC, in which a total of three weak-damping dark areas in two different ROI are shown (see Figs. 7 (a) and 8 (a)). In details, these dark areas are due to ship wake that visually are mix it up with oil in SAR image thanks to the same shape in case of tanker discharge operation. In these cases the CPD standard deviation images (Fig. 7 (b) - 8 (b)) do not show any features related to the oil look-alikes (OLA), i.e it is not possible discriminate them clearly from the surrounding sea.

\section{Conclusions}

In this study a full resolution SSC TerraSAR-X dual-polarised complex data has been processed by means of a tailored electromagnetic model for oil spill observation. The importance of this study is twofold. On one hand, it shows that dual-polarised TerraSAR-X data are right for physically based sea oil spill observation. In fact, it has been shown that using the CPD model (with no multilook technique, apart the unavoidable 3x3 window to estimate the phase difference standard deviation) is possible to highlight the presence of sea oil spills and distinguish them from weak-damping look-alikes. On the other hand, it demonstrates for the first time that X-band polarimetric data can be successfully employed to observe oil slicks, once properly modelled. 


\section{Reference}

[1] M. Migliaccio, F. Nunziata, A. Gambardella, "On The Copolarised Phase Difference for Oil Spill Observation”, Int. Journal of Remote Sensing, vol. 30, n. 6, pp. 1587-1602, 2009.

[2] F. Nunziata, A. Gambardella and M. Migliaccio, "On the Mueller Scattering Matrix for SAR Sea Oil Slick Observation”, IEEE Geosci. and Remote Sensing Letters, vol. 5, n. 4, pp. 691-695, 2008.

[3] A. Delilah, "Marine oil pollution: technologies and methodologies for detection and early warning”, European Commission JRC report, EUR 20231 EN, 2002.

[4] C. Brekke and A.H.S. Solberg, “Oil spill detection by satellite remote sensing”, Remote Sensing of Environment, vol. 95, pp. 1-13, 2005.

[5] M.F. Fingas and C.E. Brown, "Review of oil spill remote sensing”, Spill Sci. Technology Bull., vol. 4, no. 4, pp. 199-208, 1997.

[6] V. Wismann, M. Gade, W. Alpers and H. Hühnerfuss, "Radar signatures of mineral oil spills measured by an airborne multi-frequency radar”, Int. Journal of Remote Sensing, vol. 19, n.18, pp. 3607-3623, 1998.

[7] M. Eineder, T. Fritz, J. Mittermayer, A. Roth, E. Börner and H. Breit, “TerraSAR-X Ground Segment Basic Product Specification Document”, vol. Issue:1.5, TX-GS-DD-3302, 2008. 

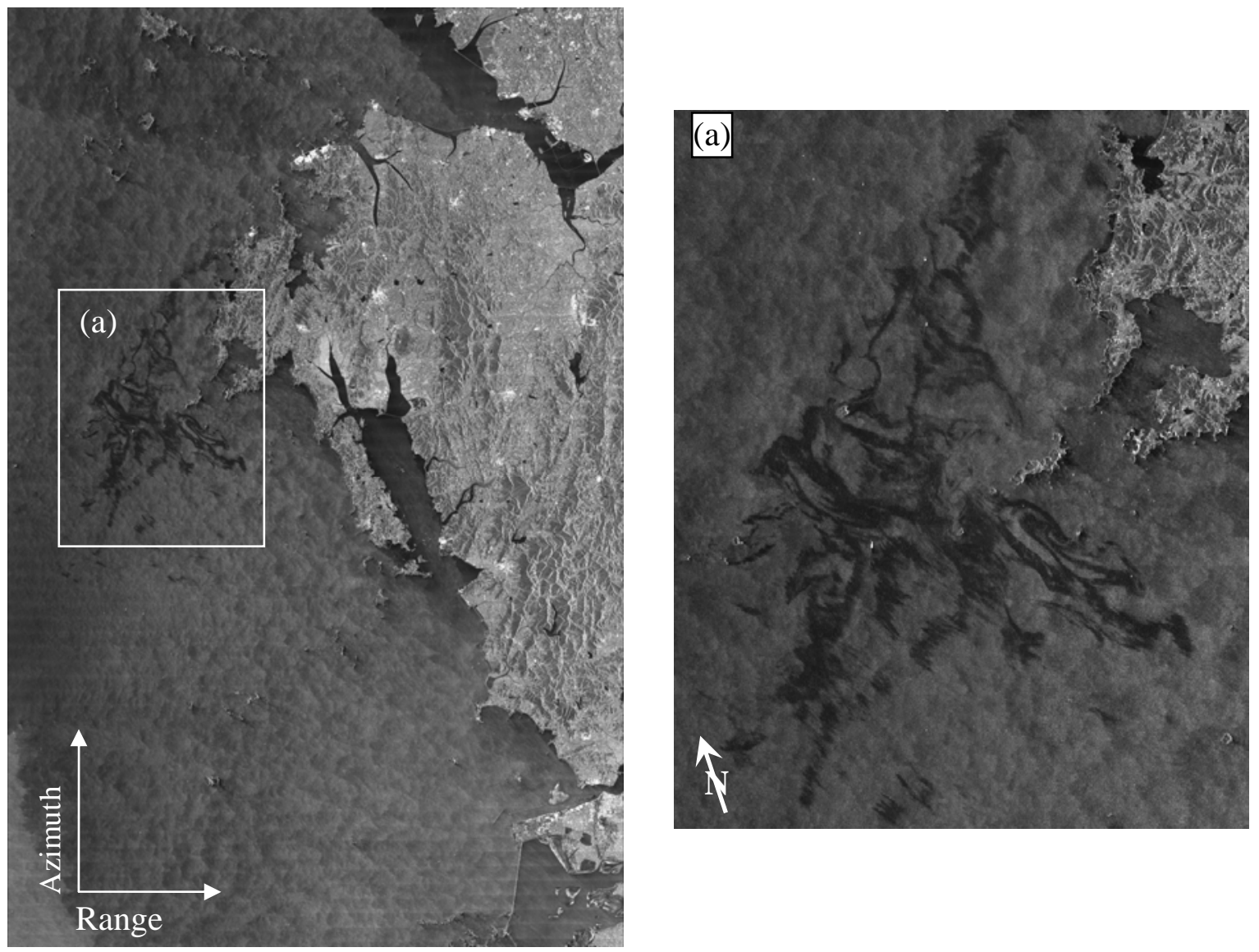

Figure 1. TSX SC VV-pol image (16 m resolution) acquired on December,12, 2007 after the Hebei Spirit tanker accident near Daesan port in South Korea (left) and details of the region of interest (right).
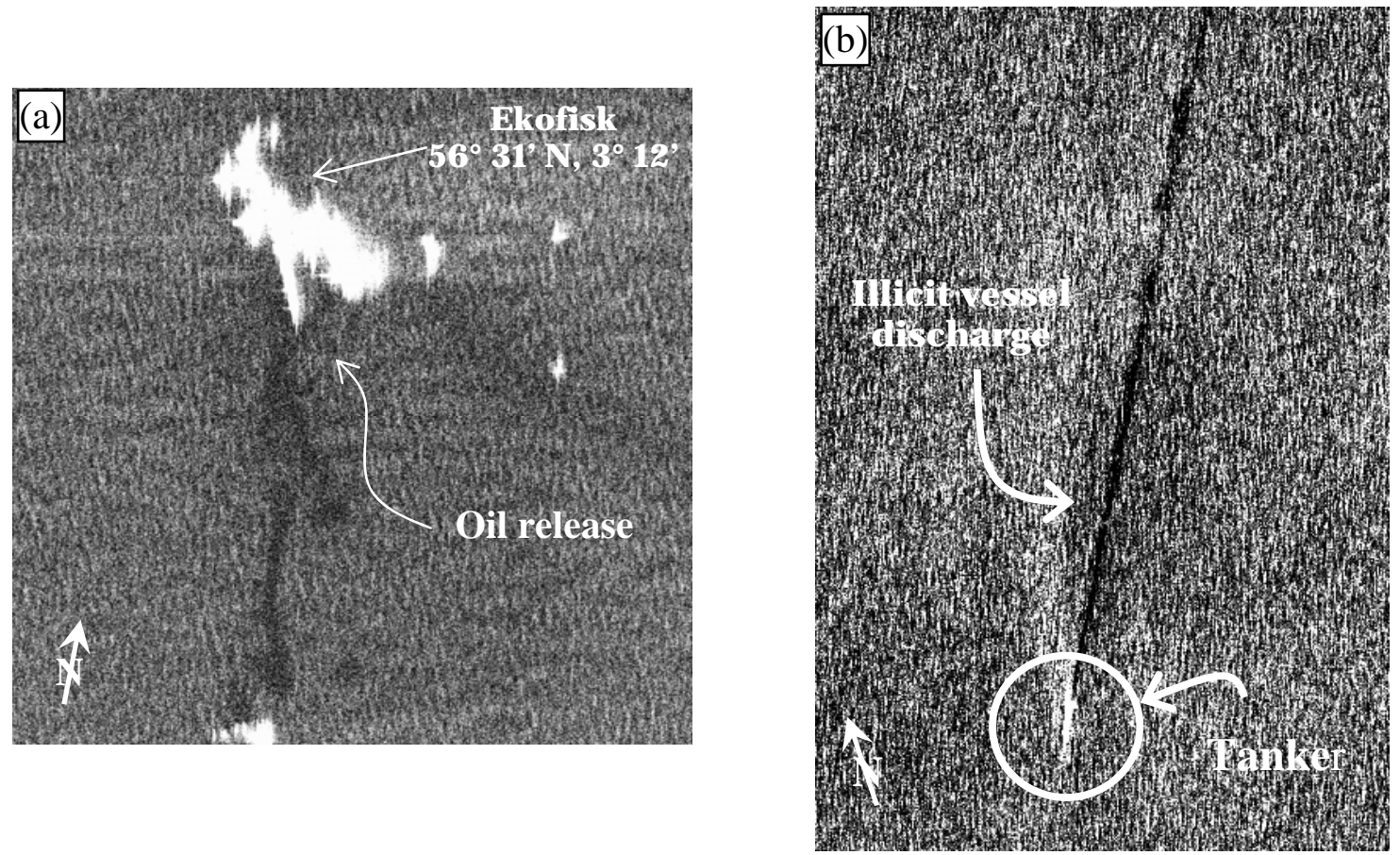

Figure 2. Two examples of operational oil spillage: (a) TSX SL VV-pol image (1.8 x 1.7 m range-azimuth resolution) acquired in Nord Sea over Ekofisk oil platform, (b) TSX SM VV-pol image (3 x 3 m rangeazimuth resolution) acquired off the Chinese East coast. 


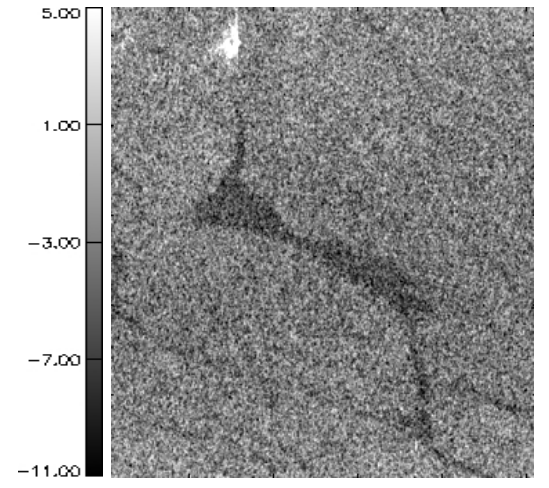

(a) VV power image $(\mathrm{dB})$

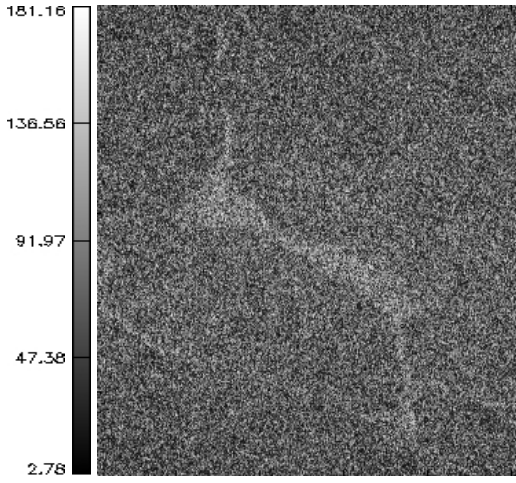

(b) CPD std image $\left(^{\circ}\right)$

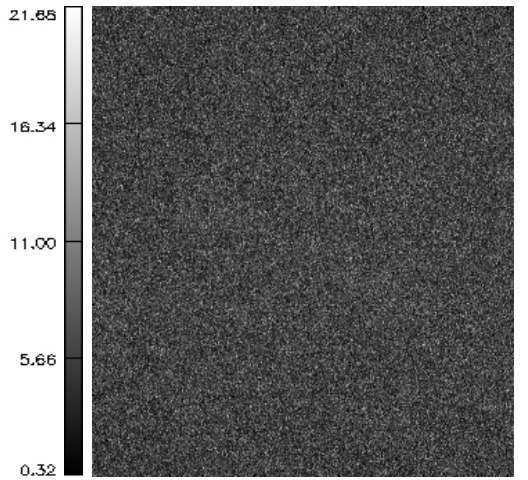

(c) CPR std image (dB)

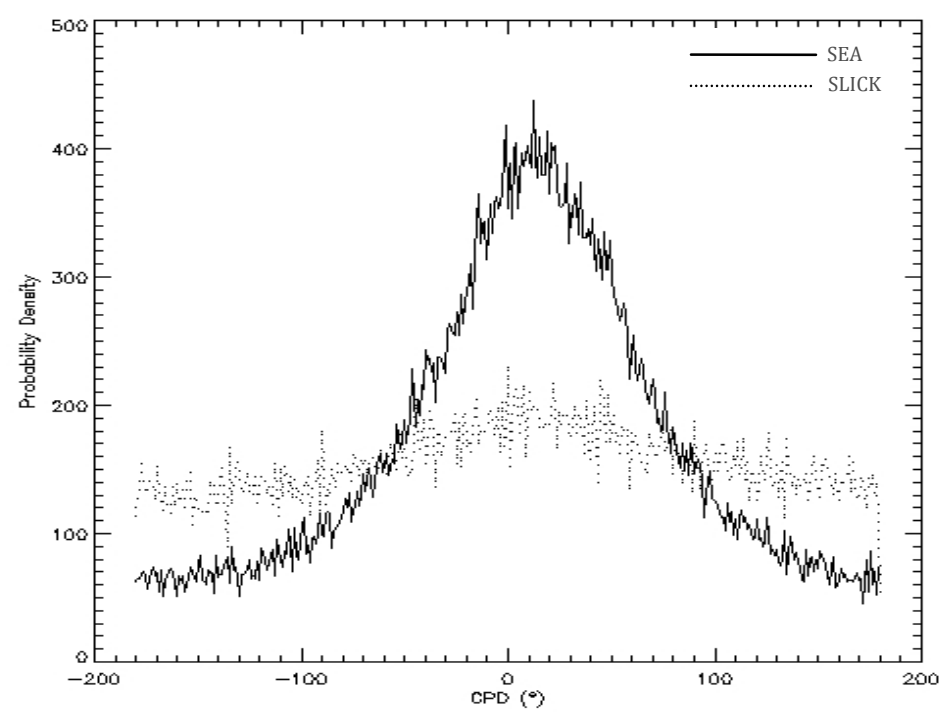

Figure 3. X-band SAR data relevant to the acquisition of 28-07-2009 at 04:18 UTC. Fig. 1(a) shows an excerpt of the X-band VV power SAR image in which an illicit vessel oil discharge is clearly visible. The estimated CPD and CPR standard deviation images are shown in grey tones in Fig. 1 (b) and 1 (c), respectively. Fig. 1 (d) shows the measured CPD pdfs relevant to both the oil covered and surrounding sea surface. 


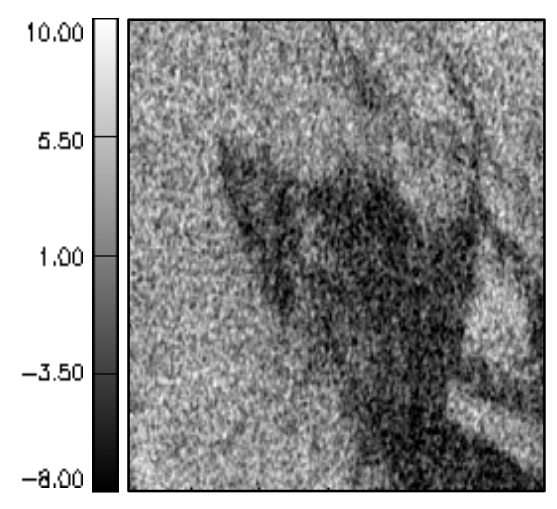

(a) VV power image $(\mathrm{dB})$

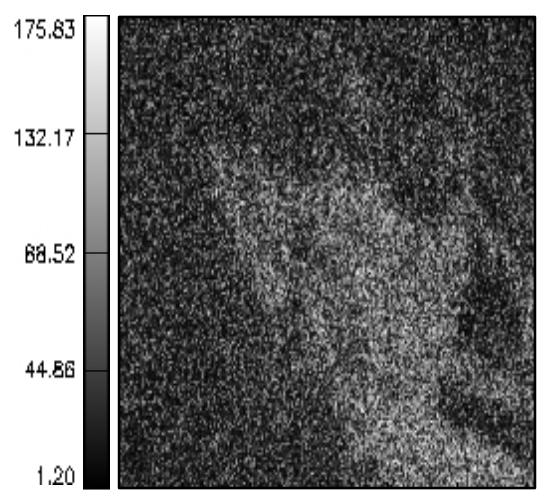

(b) CPD std image $\left(^{\circ}\right)$

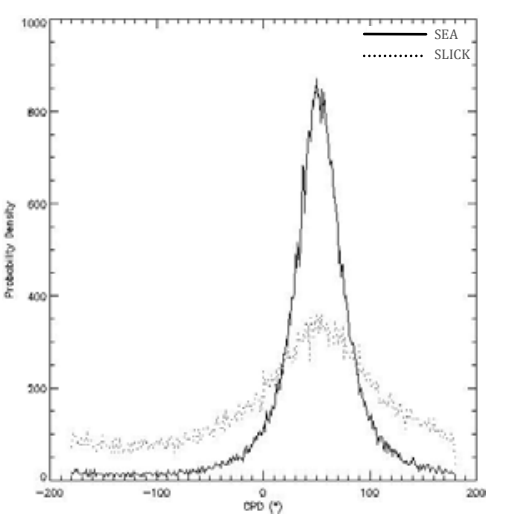

(c) Measured CPD pdfs

Figure 4. X-band SAR data relevant to the acquisition of 16-11-2007 at 03:52 UTC (North part). Fig. 2(a) shows an excerpt of the X-band VV power SAR image in which the oil spillage after the Volganeft-139 Russian oil tanker accident is clearly visible. The estimated CPD standard deviation image (grey tones) and the measured CPD pdfs are shown in Fig. 2 (b) and 1 (c), respectively.

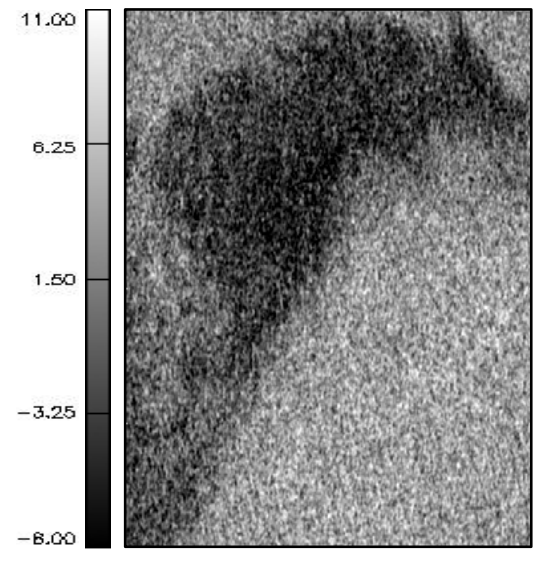

(a) VV power image $(\mathrm{dB})$

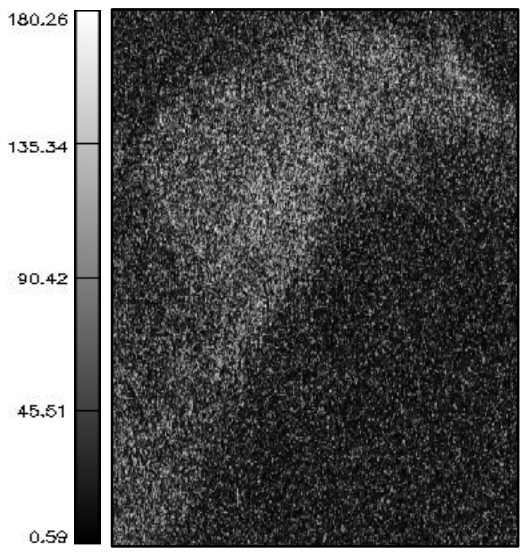

(b) CPD std image $\left({ }^{\circ}\right)$

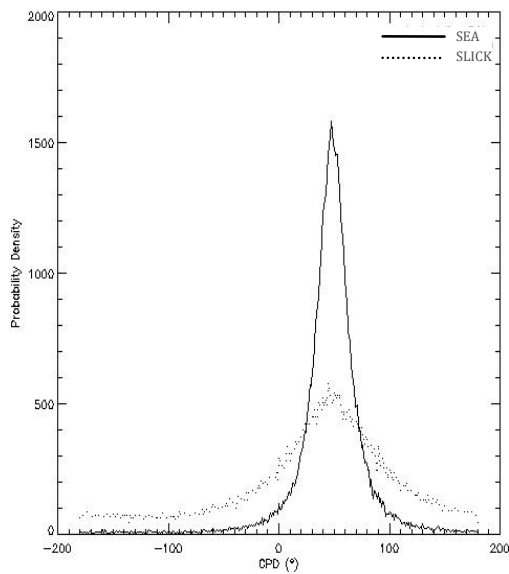

(c) Measured CPD pdfs

Figure 5. X-band SAR data relevant to the acquisition of 16-11-2007 at 03:52 UTC (Middle part). Fig. 3(a) shows an excerpt of the X-band VV power SAR image in which the oil spillage after the Volganeft-139 Russian oil tanker accident is clearly visible. The estimated CPD standard deviation image (grey tones) and the measured CPD pdfs are shown in Fig. 3 (b) and 3 (c), respectively.

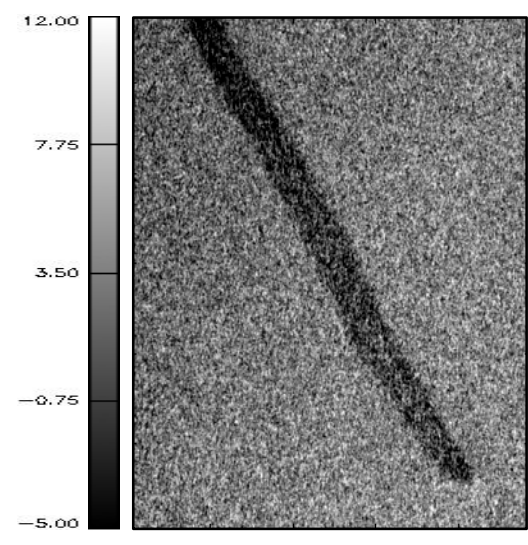

(a) VV power image $(\mathrm{dB})$

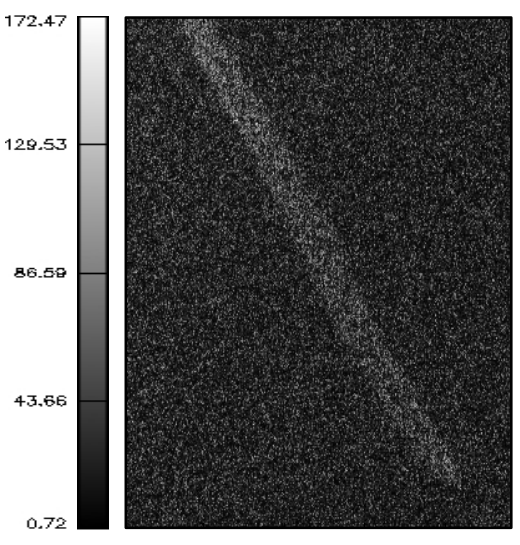

(b) CPD std image $\left(^{\circ}\right)$

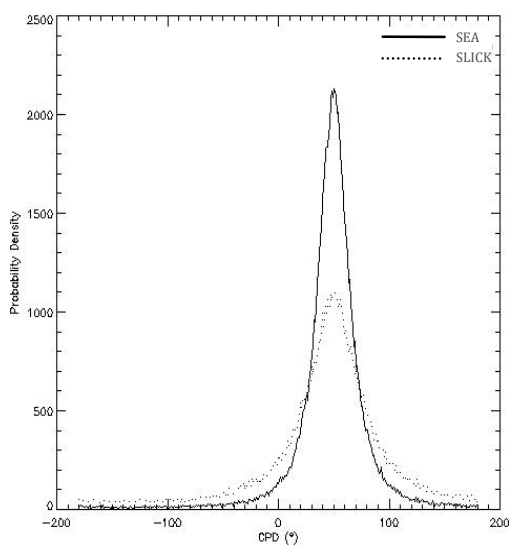

(c) Measured CPD pdfs

Figure 6. X-band SAR data relevant to the acquisition of 16-11-2007 at 03:52 UTC (South part). Fig. 4(a) shows an excerpt of the X-band VV power SAR image in which the oil spillage after the Volganeft-139 Russian oil tanker accident is clearly visible. The estimated CPD standard deviation image (grey tones) and the measured CPD pdfs are shown in Fig. 4 (b) and 4 (c), respectively. 


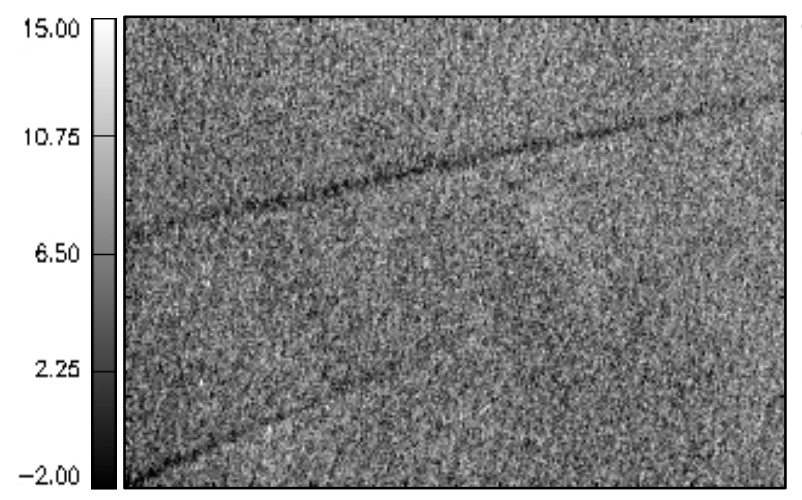

(a) VV power image (dB)

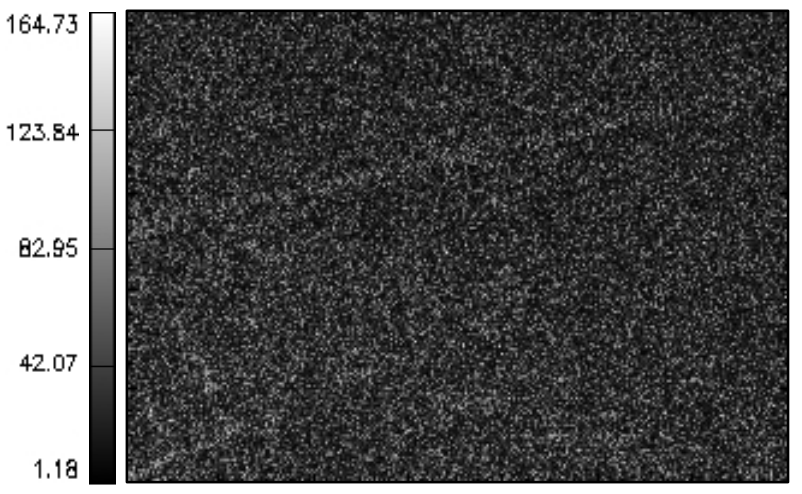

(b) CPD std image $\left(^{\circ}\right)$

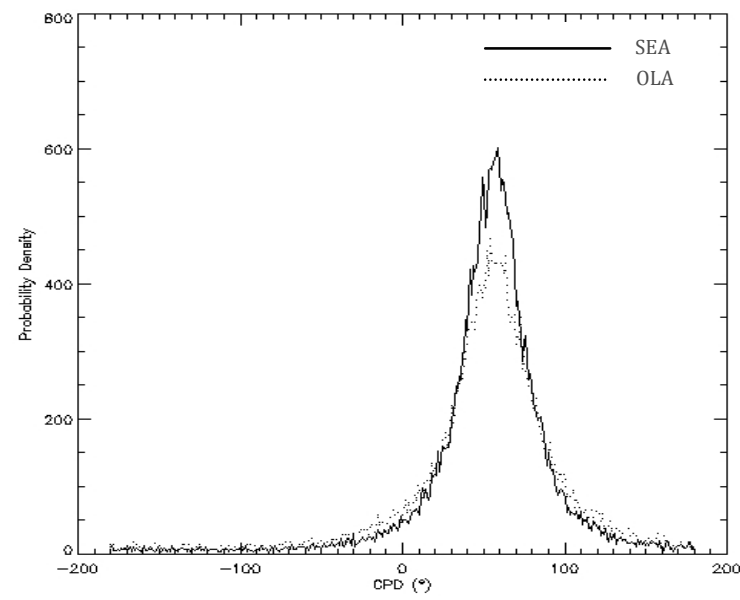

(c) Measured CPD pdfs

Figure 7. X-band SAR data relevant to the acquisition of 05-06-2009 at 05:19 UTC. Fig. 5(a) shows an excerpt of the X-band VV power SAR image in which two thin long dark areas due to ship wakes are clearly visible. The estimated CPD standard deviation image is shown in grey tones in Fig. 5 (b). Fig. 5 (c) shows the measured CPD pdfs relevant to both the oil look-alikes (OLA) area and surrounding sea surface.

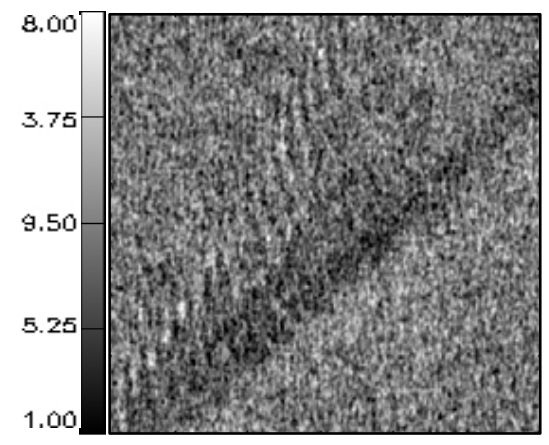

(a) VV power image $(\mathrm{dB})$

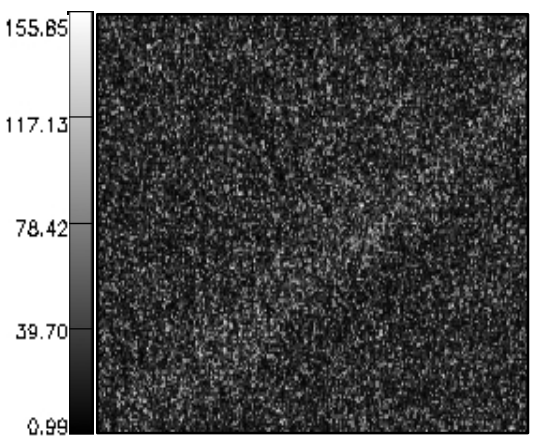

(b) CPD std image $\left(^{\circ}\right)$

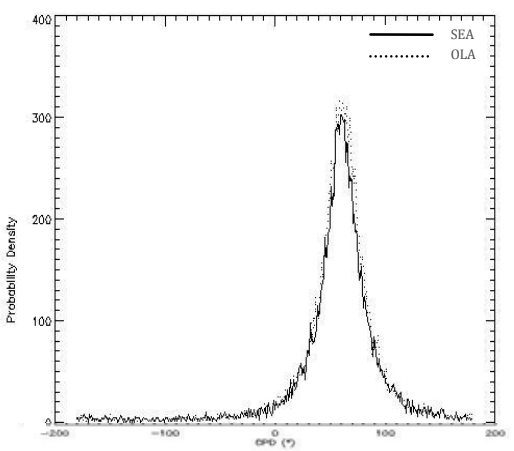

(c) Measured CPD pdfs

Figure 8. X-band SAR data relevant to the acquisition of 05-06-2009 at 05:19 UTC. Fig. 6(a) shows an excerpt of the X-band VV power SAR image in which a dark areas due to ship wake is clearly visible. The estimated CPD standard deviation image is shown in grey tones in Fig. 6 (b). Fig. 6 (c) shows the measured CPD pdfs relevant to both the oil look-alikes area and surrounding sea surface. 